\title{
Harmonic Suppression and Reactive Power Compensation Based on PWM Rectifier and SVC
}

\author{
Chunlai $\mathrm{Li}^{1}$, Yan Shi ${ }^{2}$ a , Yindong $\mathrm{Liu}^{2}$ \\ ${ }^{1}$ State Grid Qinghai Electric Power Research Institute, Xining China 810008 \\ ${ }^{2}$ School of Automation Engineering, Northeast Dianli University, Jilin China 132012 \\ aemail: 2210332595@qq.com
}

Keywords: Harmonic Suppression; Reactive Power Compensation; PWM Rectifier

\begin{abstract}
Electrolysis is an important branch of metal smelting. But due to the introduction of rectification device will produce a large number of harmonic, this undoubtedly greatly increased electric cost and the maintenance cost. This article used harmonic data in power supply system as the simulation parameters, detailed analyses static var compensation (SVC) and harmonic spectrum of PWM rectifier in the actual operation by using Simulink to build PWM rectifiers and SVC model. Through the simulation experiments to verify whether the model can better achieve inhibiting effect and enable it to reach the national standard level.
\end{abstract}

\section{The present status of the power system}

The first connection map in the power system can refer to Fig.1. The power system has stand-alone power plant, not connected to power grid of urban power supply company. Its power supply contains four sets of the same specification of generator and step-up transformer. There into, medium frequency furnace converts the power frequency into medium frequency electrotherapy from 300 to $10000 \mathrm{~Hz}$, and then smelts metals by induction heating of an induction coil. Because of using six-phase rectifier, the operational principles of harmonic generation of medium frequency furnace are the same with three-phase full bridge controlled rectifier. It will produce a large number of harmonic during the operation [1].

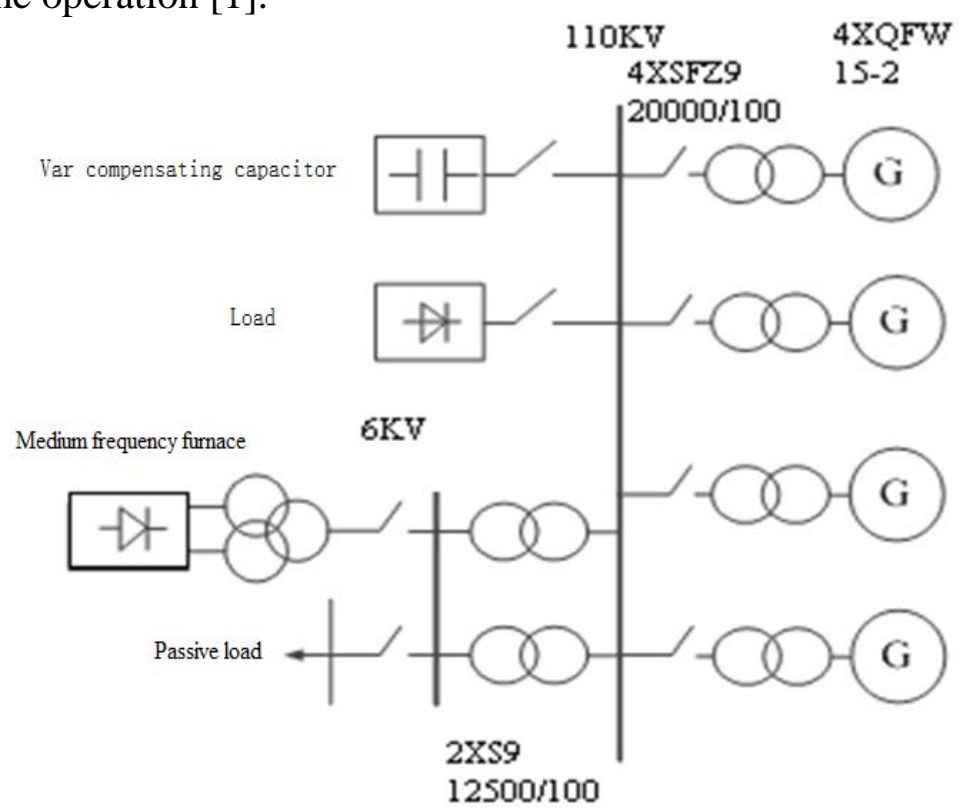

Fig.1 The first connection map in the power system

\section{Data acquisition and analysis of the power system harmonic source}

There are three large harmonic source in data acquisition of the power system, rectifier equipment, medium frequency furnace and machine works. Through analyzing the real data series of that time, it observed that the harmonic current generated by the power system is less than $1 \%$ of 
the amount of fundamental wave. At this point, the medium frequency furnace harmonic current distortion rate has reached $23.8 \%$. The main resulting cause of this gap is that the power system adopted the current domestic and foreign advanced 48-pulse rectifier transformer. Under the action of it makes the power harmonics are suppressed. However, the medium frequency furnace with 6-pulse rectifier transformer produces larger harmonic current and high distortion rate [2].

The actual running tests found that the internal electrical equipment is mainly affected by the harmonic produced by medium frequency furnace. To meet the needs of the whole test, we can convert harmonic current of medium frequency furnace into $380 \mathrm{~V}$ side. At this point, each harmonic current are 141.9A, 87.63A, 47.6A, 35.5A, 17.4A, 12.8A for 5th, 7th, 11th, 13th, 17th and 19th harmonics current respectively. The total harmonic current distortion rate is $25.2 \%$. Due to the harmonics in public supply network, allowable value of the harmonic current injected into the public connection is not prescribed in view of the $575 \mathrm{~V}$ voltage standard. We might as well contrasted 380V power grid harmonic current value. Thereinto, the 5th, 7th, 11th, 13th harmonics current are more than 1.71 times, 1.5 times, 1.29 times and 1.11 times respectively. The harmonic current generated by the medium frequency furnace in practical operation data are shown in Table 1. In the operation of the whole power system, it can be found harmonics produced by medium frequency furnace impact on system greatly.

Table 1 The harmonic current generated by the medium frequency furnace

\begin{tabular}{|c|c|c|c|c|c|c|c|c|c|c|}
\hline $\begin{array}{l}\text { Percentage } \\
\text { content }\end{array}$ & Harmonic number & 1 & 5 & 7 & 11 & 13 & 17 & 19 & 35 & 37 \\
\hline \multirow{2}{*}{$23.80 \%$} & Electricity/ kA & 1.81 & 0.34 & 0.21 & 0.11 & 0.08 & 0.04 & 0.03 & 0.02 & 0.02 \\
\hline & Percentage content/\% & 100 & 18.9 & 11.6 & 6.3 & 4.7 & 2.3 & 1.7 & 1.1 & 0.8 \\
\hline \multirow{3}{*}{$10.60 \%$} & Harmonic number & 1 & 5 & 7 & 11 & 13 & 17 & 19 & 35 & 37 \\
\hline & Voltage /V & 360.9 & 16.3 & 11 & 13.9 & 12.3 & 13 & 12 & 12.7 & 7.2 \\
\hline & Percentage content/\% & 100 & 4.5 & 3.2 & 3.9 & 3.6 & 3.6 & 3.3 & 3.5 & 2 \\
\hline
\end{tabular}

\section{Establish harmonic suppression simulation model of the power system}

To fully realize the whole system simulation process is consistent with the actual operation process of the basic conditions, the system parameters, harmonic source and the choice of modules used in the model building are becoming especially important [3]. According to the existing material, component parameters and system impedance are selected and then set up the simulation system combined with the above parameters. The harmonic suppression simulation model is established under the Simulink, which can be divided into harmonic source module, rectifier filter module and the power system circuit module. The AC power using encapsulation function is encapsulated in "AC current source". Due to main function of the harmonic source in the system is to produce harmonic current, and only relates to its working condition and the voltage, has smaller influence on the impedance of the whole system. So the harmonic source is equivalent to a constant current source and its internal resistance is infinite [4].

Based on the relevant data from the field power plant (Table 1), the AC current source in the Simulink model is used in the system, so as to obtain the harmonic source module (shown in Fig.2). Fig.2 shows the specific composition of the harmonic source module. As can be seen from the Fig.2, harmonic source is composed of nine AC current source, and the value of each branch circuit corresponds to the each harmonic current value. In addition, There is a very important element in the harmonic source module, in parallel with a 1000 Ohms resistor. The internal resistance of the constant current source is infinite, but the AC current source used in this simulation environment in the component library is no internal resistance. According to the analysis of the literature materials, the harmonic source can not work in the condition of no internal resistance. So the resistance is especially important. The simulation model of the whole system can refer to Fig.3 [5][6]. 


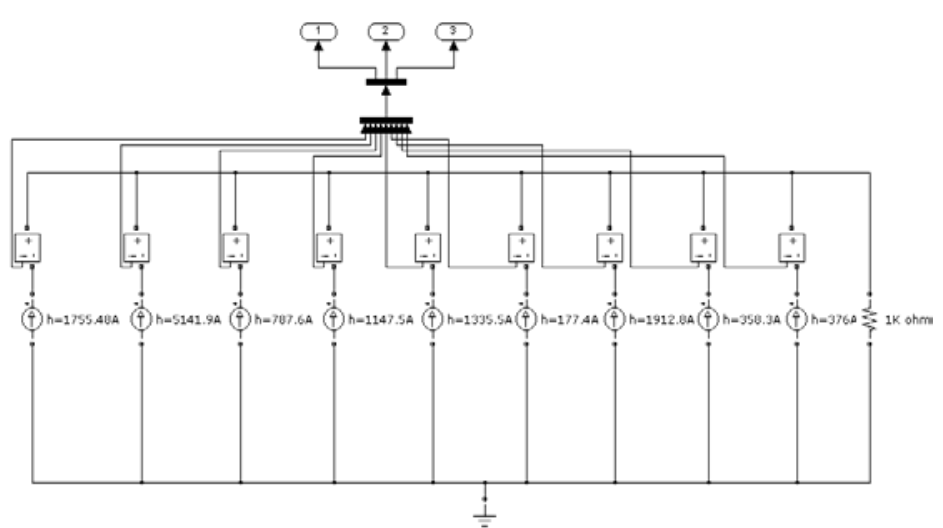

Fig.2 Harmonic source simulation model

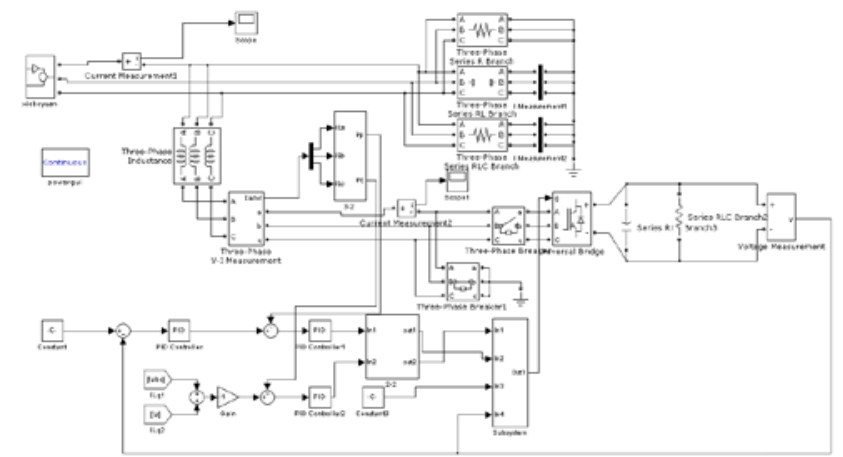

Fig.3 The simulation model of the whole system

\section{Harmonic simulation results and analysis of the power system}

Fig. 4 is the comparison result of the simulation of the operating system before and after the filter. Known from the figure, the rectifier starts working at 0.5s. At this time, the harmonic voltage are time-variant and the waveform is relatively significant improvements, and more close to the sine. Though still because of the limitations of currently used technology, harmonic failed to filter completely and there is the phenomenon of irregular sine. Fig. 5 is the variation of current through the rectifier before and after the PWM rectifier which starts working at $0.5 \mathrm{~s}$. It is not difficult to see that the current waveform of the rectifier that PWM rectifier has not started is completely ideal. According to the design principle, the rectifier began to produce current quantity used to suppress the power harmonics after the PWM rectifier is ready for use at 0.5s [7].

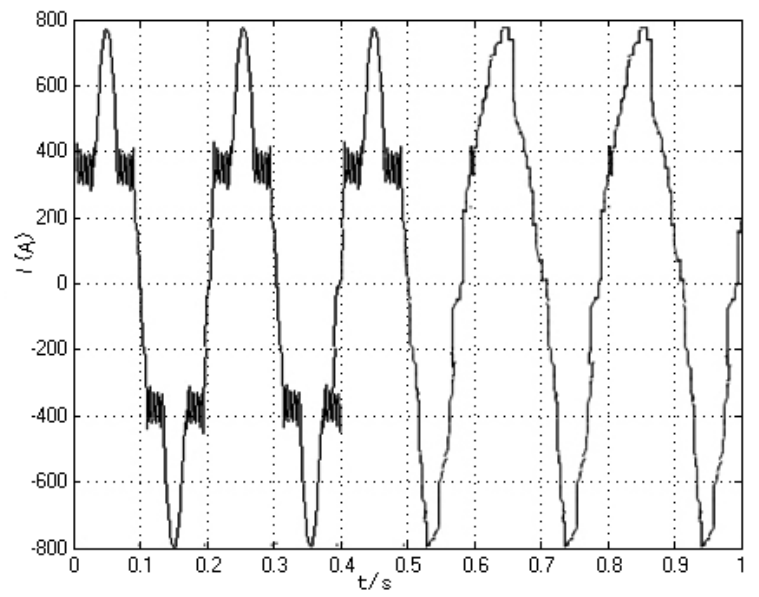

Fig.4 Voltage before and after the filter

According to the simulation results, we can analyse harmonic wave in the power grid. Concrete analysis as shown in Fig.6 and Fig.7. Among them, Fig.6 showed the voltage waveform without harmonic suppression before $0.05 \mathrm{~s}$, Fig.7 showed the voltage waveform with harmonic suppression after $0.05 \mathrm{~s}$ [8][9]. 


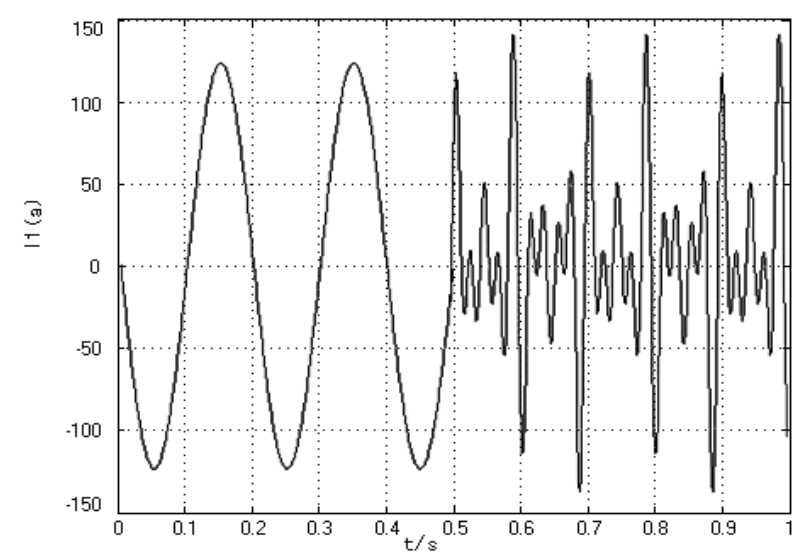

Fig.5 The variation of current through the rectifier before and after the PWM rectifier

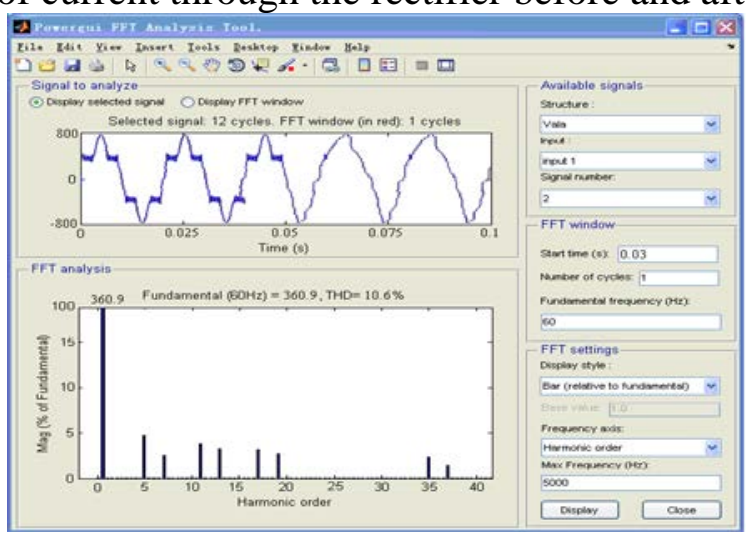

Fig.6 Frequency spectrum analysis before the harmonic treatment

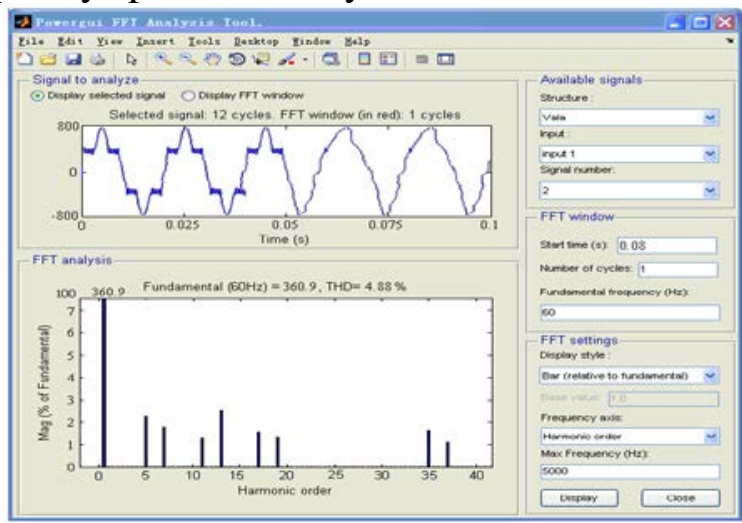

Fig.7 Frequency spectrum analysis after the harmonic treatment

Fig. 6 showed voltage amplitude is $360.9 \mathrm{~V}$, but the total harmonic distortion value-10.6\% before harmonic suppression-is more than two times the harmonic voltage distortion rate limit beyond to the request of GB(GBT/14549-93). We can see from Fig.7 that the total harmonic distortion value after the harmonic suppression processing is in line with national standards of only $4.88 \%$. This further proves this experiment simulation system has the characteristics of reasonable and reliable.

\section{The estimation of economic benefit on reactive compensation and harmonic suppression}

Harmonic current in the circuit will shorten the using lifetime of equipment. When the harmonic current exceeds nominal standard, the more the shorter the life of the device, causing an indirect equipment depreciation loss. Generally by the installation of harmonic suppression equipment, a year can save $20 \%$ 30\% of the equipment maintenance and depreciation expense. In addition, the harmonic in the power circuit and power equipment will cause additional copper loss and iron loss of the lines and equipment, resulting in the extra power consumption. The existence of harmonic voltage will cause harmonic distortion power loss. Assuming that harmonic distortion power before 
and after the harmonic suppression is $P_{J 1}, P_{J 2}$ respectively, power factor is $\cos \varphi$ and its economic benefit is $C_{P}$ :

$$
C_{P}=P_{J 1}-P_{J 2}
$$

By the calculation, $P_{J 1}=38.26 \cos \varphi k W, P_{J 2} \approx 0.46 P_{J 1}$

$$
C_{P}=P_{J 1}-P_{J 2} \approx 0.44 P_{J 1}=16.38 \cos \varphi k W
$$

Set the equipment running time for each year is $H_{y}$ hours, electricity price is $C_{D} ¥ / \mathrm{Kwh}$, the benefits for each year

$$
\begin{aligned}
& C_{Y}=C_{P} H_{y} C_{D} \approx 16.83 \cos \varphi H_{y} C_{D} \\
& \text { If } H_{y}=365 \times 22=8033 \text { hours, } C_{D}=0.35 ¥ / \text { Kwh, } \cos \varphi=1 \\
& C_{Y}=C_{P} H_{y} C_{D} \approx 16.83 \times 8033 \times 0.35 \times 0.6 \approx 47300
\end{aligned}
$$

\section{Conclusion}

The model of medium frequency furnace is established by combining the actual situation of the power system. And then analyze related harmonic data for the simulation system. And the results of computer simulation indicate that this system can improve harmonics in the operating system, better to control the voltage harmonic distortion rate within the scope of the national standard, and extend the service life of equipment and reduce the maintenance cost, bringing high economic benefits.

\section{Acknowledgement}

This study was supported by QingHai Province Key Laboratory of Photovoltaic grid connected power generation technology (Grant No. SGQHDKYODWJS1500037).

\section{References}

[1] Jun Peng, Zhong-dong Yin, et al. The research status of static var compensator [J]. Sichuan Electric Power Technology, 2006, 29(3):26-28.

[2] Yong Zhang. Study on the harmonic in the power network of Shandong Dong Yue energy corporation aluminum factory [D]. Jinan: Shandong University of Science and Technology, 2005.

[3] Zheng-shi Wang, Hui-Ming Chen. Simulation Research on a Novel Rectifier and Its Control Strategies with Functions of Reactive Power Compensation and Harmonic Current Filtering [J]. Converter Technology \& Electric Traction, 2007, (1):36-39.

[4] C A Munoz. A New High-power-factor Three-phase AV-DC Converter: Analysis Design and Experimentation. IEEE Transactions on Power Electronics, 1999, 14(1):90-97.

[5] Chang-jiang Zhan, Quan-hua Qin, et al. Study on mathematical model and system simulation of the three-level PWM reversible rectifier [J]. Proceedings of the CSEE, 1999, 19(7):45-48.

[6] Ekanayake J B, Jenkins M. A three-level advanced static VAr compensator [J]. IEEE Transactions on Power Delivery, 1996, 11(1):540-545.

[7] Ke-yuan Huang, Yi-kang He, et al. Study of three-phase PWM rectifier for AC-excitation [J]. Journal of Zhejiang University(Engineering Science), 2004, 38(1):48-51.

[8] Schauder C, Mehta H. Vector analysis and control of advanced static VAR compensators [C]// International Conference on Ac \& Dc Power Transmission. 1993:299-306.

[9] M. Depenbrock. Direct self-control (DSC) of inverter-fed induction machine [J]. IEEE Transactions on Power Electronics, 1988, 3(4):420-429. 